\title{
Hybrid Medium Access Control for Time-switching Simultaneous Wireless Information and Power Transfer
}

\author{
Yong-Seong Kim, ${ }^{1,3}$ Jung-Hyok Kwon, ${ }^{2}$ Yongseok Lim, ${ }^{3}$ \\ Eui-Jik Kim, ${ }^{4}$ Dongwan Kim, ${ }^{5 *}$ and Yong Sin Kim ${ }^{1 * *}$ \\ ${ }^{1}$ School of Electrical Engineering, Korea University, \\ 145 Anam-ro, Seongbuk-gu, Seoul 02841, South Korea \\ ${ }^{2}$ Smart Computing Laboratory, Hallym University, \\ 1 Hallymdaehak-gil, Chuncheon, Gangwon-do 24252, South Korea \\ ${ }^{3}$ Smart Network Research Center, Korea Electronics Technology Institute, \\ 11 World Cup buk-ro 54-gil, Mapo-gu, Seoul 03924, South Korea \\ ${ }^{4}$ School of Software, Hallym University, \\ 1 Hallymdaehak-gil, Chuncheon, Gangwon-do 24252, South Korea \\ ${ }^{5}$ Department of Electronic Engineering, Dong-A University, \\ 37 Nakdong-Daero 550beon-gil, Saha-gu, Busan 49315, South Korea
}

(Received April 21, 2019; accepted August 14, 2019)

Keywords: batteryless IoT, hybrid MAC, superframe structure, TS-SWIPT, WPT

This paper presents hybrid medium access control (MAC) for time-switching simultaneous wireless information and power transfer (TS-SWIPT) that improves channel utilization and the timeliness of data and power delivery. To this end, the proposed hybrid MAC uses a superframe structure including a beacon period (BP), a contention access period (CAP), and a channel time allocation period (CTAP), and adaptively adjusts the CAP and CTAP lengths according to the data and power delivery time requested by sensor devices. Furthermore, it adjusts the priority of data and power delivery according to the residual power level of each sensor device. To evaluate the performance of the proposed hybrid MAC, an experimental simulation was conducted. The results show that the proposed hybrid MAC outperforms the existing MAC in terms of resource utilization, aggregate throughput, and charging delay.

\section{Introduction}

Recently, with the rapid advance of information and communication technology (ICT), the Internet of Things (IoT) has been applied to a wide range of applications such as smart homes, connected cars, smart healthcare, and factory automation. ${ }^{(1,2)}$ In such applications, a number of sensor devices are generally deployed to detect changes in the surrounding environment and to transmit the sensing data to the intended destination. ${ }^{(3)}$ However, these sensor devices typically operate with a battery having a limited power source. This results in high maintenance costs for battery replacement and a short device lifetime. ${ }^{(4)}$ Therefore, for cost-efficient and reliable IoT applications, it is essential to provide continuous and sufficient power to the sensor devices without replacing their battery.

\footnotetext{
*Corresponding author: e-mail: dongwankim@dau.ac.kr

** Corresponding author: e-mail: shonkim@korea.ac.kr

https://doi.org/10.18494/SAM.2019.2503
} 
In recent years, there has been growing interest in batteryless IoT that can supply power to devices via energy harvesting (EH) technology, which converts the collected ambient signals into a direct current (DC) voltage. ${ }^{(5)}$ For the realization of batteryless IoT, the use of radio frequency (RF)-based wireless power transfer (WPT) technology has been widely considered since it allows devices to collect power from the intentionally transmitted RF signals. ${ }^{(6)}$ In particular, simultaneous wireless information and power transfer (SWIPT), which uses RFbased WPT technology, is regarded as a core technology for realizing batteryless IoT. This is because SWIPT has the capability to charge devices while maintaining information exchange.

SWIPT can be categorized into the following two types depending on the architecture of the receiver: time-switching SWIPT (TS-SWIPT) and power-splitting SWIPT (PS-SWIPT). In both types, the receiver contains information decoding (ID) and EH circuits, but the RF signal partitioning operation for ID and EH circuits is different for each type. Specifically, in TSSWIPT, the receiver antenna switches between ID and EH circuits according to the specified period. On the other hand, in PS-SWIPT, the receiver splits the received RF signal into power and information streams, and transmits each of them to the corresponding circuit. In this paper, we consider TS-SWIPT. TS-SWIPT has a simpler hardware architecture than PS-SWIPT, which is advantageous for implementation.

In TS-SWIPT, the network performance, including the throughput and device lifetime, varies greatly depending on when and for how long the receiver performs ID and EH. In other words, for reliable TS-SWIPT, efficient medium access control (MAC) that can satisfy the following requirements is essential.

- High resource utilization: The RF signals for both ID and EH are transmitted through limited wireless resources. Therefore, the RF signal for EH should be transmitted only when necessary, and unnecessary delay caused by control packet exchange should be minimized.

- Traffic-type adaptation: The time required for data delivery depends on the data size and supported data rate, but that for power delivery depends on the transmission power (Tx power) and the distance between the devices. Therefore, resource allocation should be conducted separately according to the traffic type.

- Timely power delivery: To prevent the power depletion of devices, the priority of power delivery should be determined according to the residual power level of each device. In addition, a deterministic delay in power delivery should be supported.

Unfortunately, to the best of our knowledge, dedicated MAC for TS-SWIPT has not yet been developed. Therefore, there is a need to develop MAC for TS-SWIPT that can satisfy the above requirements. For TS-SWIPT, the existing IEEE 802.11 MAC standard can be utilized. ${ }^{(7)}$ However, since it uses carrier sense multiple access with collision avoidance (CSMA/CA), resource utilization can be low. Also, it is difficult to support efficient power delivery because it only considers data delivery and does not support priority control. To overcome this problem, the RF-MAC proposed by Naderi et al. can be applied to TS-SWIPT. In RF-MAC, only a device with power lower than a certain level is allowed to charge its power and priority control based on the residual power level of each device is provided. ${ }^{(8)}$ However, RF-MAC uses contention-based multiple access, and it is difficult to guarantee the timeliness of power delivery. For timely power delivery, Iannello et al. investigated centralized scheduled MAC for 
WPT. $^{(9)}$ This allows the device to transmit power in a timely manner utilizing dedicated slots. However, it may cause a long delay for resource allocation. Therefore, it is difficult to meet the requirements of TS-SWIPT.

In this paper, we propose hybrid MAC for TS-SWIPT. To improve channel utilization and the timeliness of data and power delivery, the proposed hybrid MAC uses a superframe structure including a beacon period (BP), a contention access period (CAP), and a channel time allocation period (CTAP), which supports both contention-based and scheduled multiple access. It adaptively adjusts the CAP and CTAP lengths and the priority of data and power delivery. The CTAP consists of multiple power slots (PSLOTs) and data slots (DSLOTs). Each PSLOT length is determined by considering the distance between the hybrid access point (HAP) and the device, the Tx power of the HAP, and the charging power level of each sensor device. Each DSLOT length is determined by considering the supported data rate and data size. The superframe duration except the CTAP length is used as the BP and CAP since the superframe duration is fixed. The priority of data and power delivery is determined according to the residual power level of each sensor device. The simulation results show that the proposed hybrid MAC exhibits higher performance than the existing MAC with respect to resource utilization, aggregate throughput, and charging delay.

The rest of this paper is organized as follows. A detailed description of hybrid MAC for TSSWIPT is presented in Sect. 2. The performance evaluation is presented in Sect. 3. Finally, Sect. 4 concludes the paper.

\section{Hybrid MAC for TS-SWIPT}

In this paper, we consider a star topology network consisting of a single HAP and multiple sensor devices. All devices are equipped with a single omnidirectional antenna with a fixed transmission range. The HAP allocates wireless resources for data exchange and power charging, referring to the requests received from sensor devices, in a centralized manner. The power is only transmitted from the HAP to the sensor devices, and the data can be transmitted from the HAP and sensor devices. The HAP and sensor devices operate in a half-duplex mode; thus, uplink and downlink communications do not occur simultaneously.

Figure 1 shows the superframe structure of the proposed hybrid MAC for TS-SWIPT. The superframe structure is composed of the BP, CAP, and CTAP. The beacon is used to announce the presence of a network and to synchronize the sensor devices. Through the BP, the HAP periodically broadcasts the beacon frame. The CAP is divided into the regular and notification CAPs. In the regular CAP, the CTAP allocation request message is transmitted from the sensor devices to the HAP using contention-based CSMA/CA. The length of the regular CAP varies with the CTAP length. In other words, the longer the CTAP, the shorter the CAP. The notification CAP is used to notify the index and length of PSLOTs and DSLOTs. The CTAP is a dedicated period for power and data delivery. This period consists of the power and data CTAPs, each of which is divided into multiple PSLOTs and DSLOTs, respectively. The numbers of PSLOTs and DSLOTs in a superframe can be different since the sensor devices selectively request PSLOTs or DSLOTs as needed. The lengths of PSLOTs and DSLOTs are 


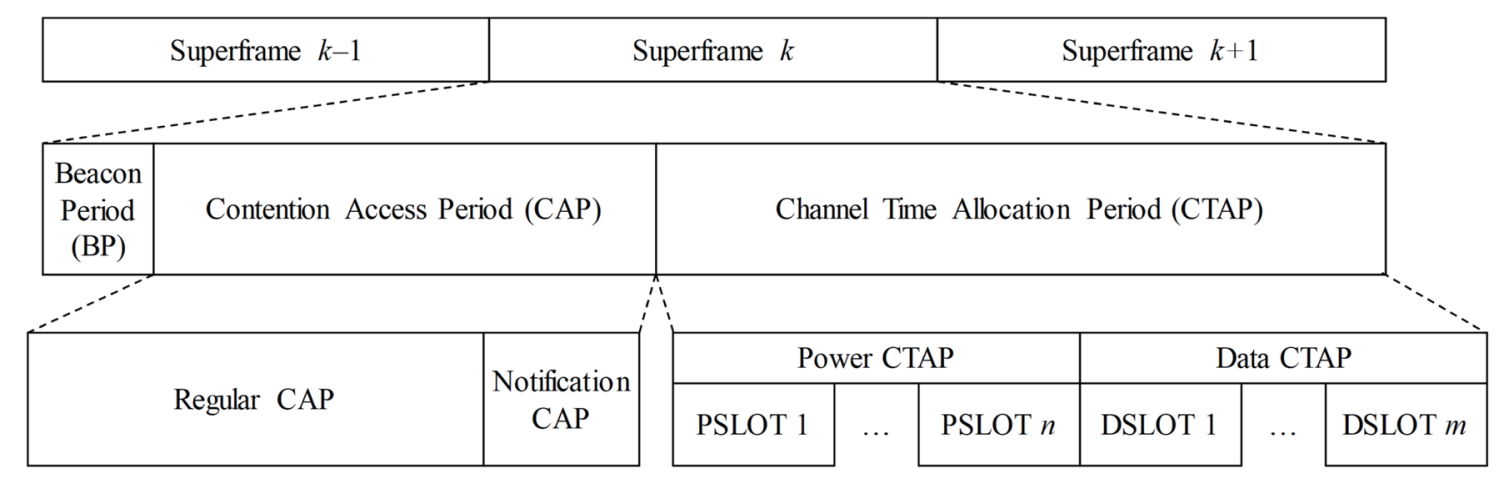

Fig. 1. Superframe structure.

adaptively determined according to the network environment and the information in the CTAP allocation request message.

Figure 2 shows the overall operation of the hybrid MAC for TS-SWIPT. During the CAP, the sensor devices attempt to transmit the CTAP allocation request message to the HAP using CSMA/CA. The CTAP allocation request message includes the charging power level, data size, and residual power level. The charging power level and data size are used to calculate the PSLOT and DSLOT lengths, respectively. The residual power level is used for priority determination. Upon receiving the CTAP allocation request message, the HAP calculates the CTAP length and transmits an acknowledgment (Ack) to inform the start time of the notification $\mathrm{CAP}\left(\mathrm{ST}_{\mathrm{NCAP}}\right)$ in the superframe and the successful reception of the CTAP allocation request message. $\mathrm{ST}_{\mathrm{NCAP}}$ is updated whenever the CTAP length changes. The other sensor devices obtain $\mathrm{ST}_{\mathrm{NCAP}}$ by overhearing the Ack. At the beginning of the notification CAP, the HAP determines the priority of data and power delivery by considering the residual power level of each sensor device. Then, it broadcasts the CTAP allocation notification message to notify the allocation information for PSLOTs and DSLOTs (i.e., the index and length of the PSLOT and DSLOT for each sensor device). Note that, in the figure, TxOffset is the waiting time used for the reception preparation and guard time. The HAP transmits power to the sensor device during the allocated PSLOT, and the HAP and sensor device exchange data during the allocated DSLOT. In the CTAP, except for the allocated PSLOT and DSLOT, the sensor device enters the sleep mode.

As mentioned earlier, the CAP length is adaptively adjusted and $\mathrm{ST}_{\mathrm{NCAP}}$ is the end time of the regular CAP. Algorithm 1 shows the $\mathrm{ST}_{\mathrm{NCAP}}$ determination procedure. In the algorithm, the HAP repeatedly determines and updates $\mathrm{ST}_{\mathrm{NCAP}}$ whenever it receives the CTAP allocation request message from the sensor device during the regular CAP. The HAP initializes the variables (i.e., $\mathrm{T}_{\text {Current }}, \mathrm{ST}_{\mathrm{NCAP}}$, and $\mathrm{L}_{\mathrm{CTAP}}$ ). $\mathrm{T}_{\text {Current }}$ denotes the current time, which is incremented in each slot time unit from the beginning of each superframe. $\mathrm{T}_{\text {Current }}$ is measured by the notification timer of the HAP. $\mathrm{ST}_{\mathrm{NCAP}}$ is initialized to $\mathrm{L}_{\text {Superframe, which }}$ is the superframe duration fixed by the HAP. $\mathrm{ST}_{\mathrm{NCAP}}$ decreases as the CTAP length $\left(\mathrm{L}_{\mathrm{CTAP}}\right)$ increases. $\mathrm{L}_{\mathrm{CTAP}}$ is initially set to zero and increases when each PSLOT or DSLOT is newly 


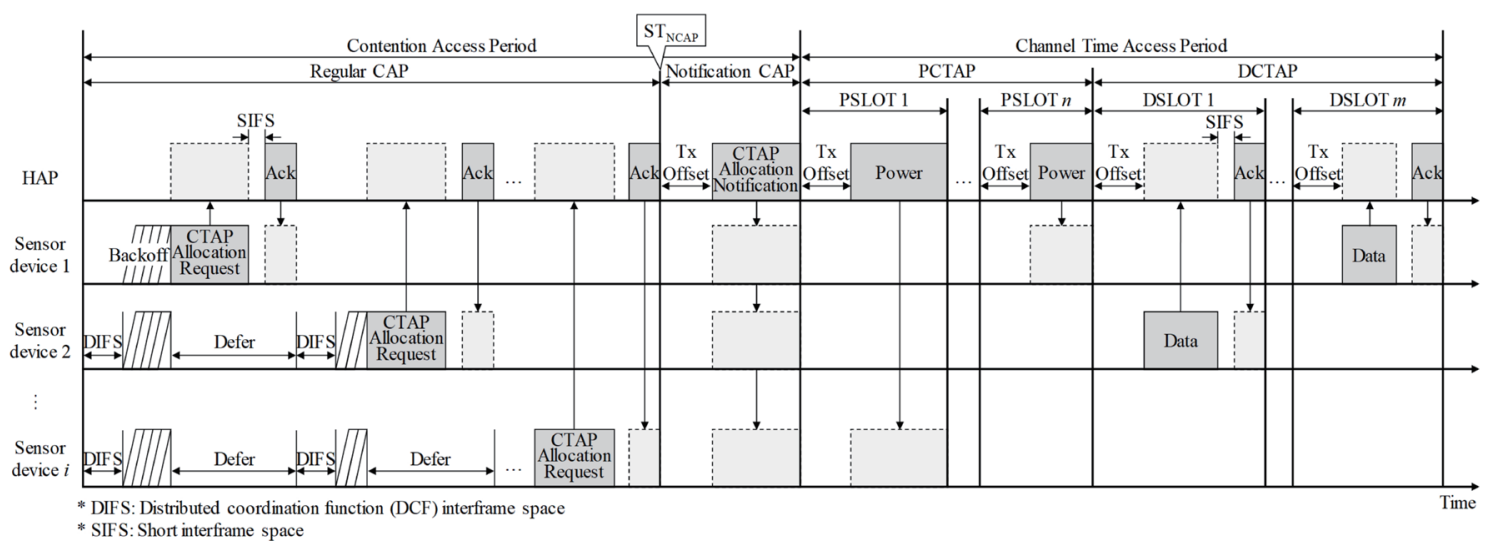

Fig. 2. Overall operation of hybrid MAC for TS-SWIPT.

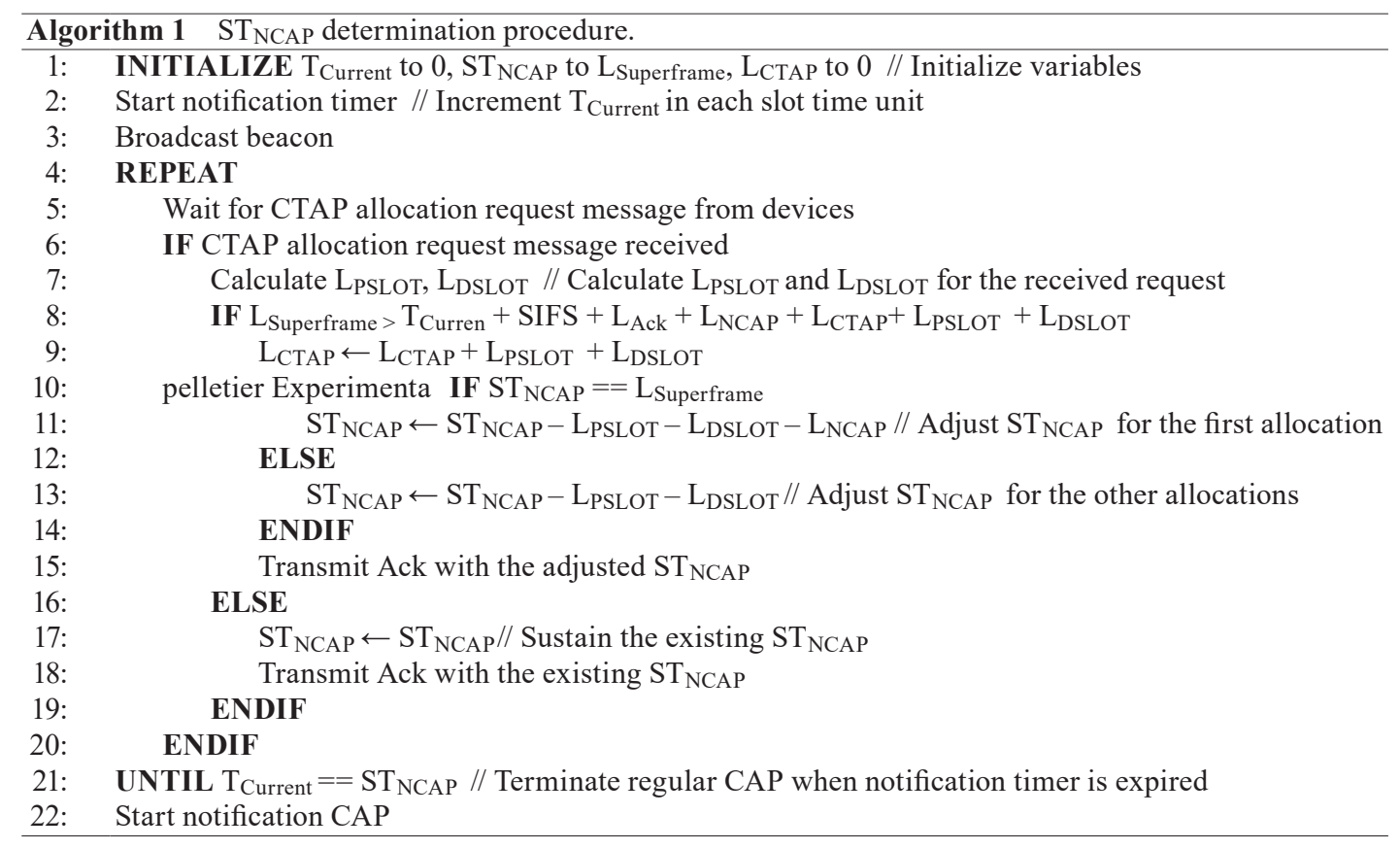

allocated. If a CTAP allocation request message is received, the HAP examines whether the requested length of each PSLOT (LPSLOT) or DSLOT (LDSLOT) can be accommodated within the remaining length of the superframe duration. If possible, the HAP decreases $\mathrm{ST}_{\mathrm{NCAP}}$ by the sum of $\mathrm{L}_{\mathrm{PSLOT}}$ and $\mathrm{L}_{\mathrm{DSLOT}}$. Otherwise, the HAP maintains the existing $\mathrm{ST}_{\mathrm{NCAP}}$. In this case, the requested PSLOT and DSLOT are not allocated for the CTAP. Note that $\mathrm{ST}_{\mathrm{NCAP}}$ decreases by the sum of the notification CAP length $\left(\mathrm{L}_{\mathrm{NCAP}}\right)$, $\mathrm{L}_{\mathrm{PSLOT}}$, and $\mathrm{L}_{\mathrm{DSLOT}}$ when the HAP first allocates the PSLOT and DSLOT. When $\mathrm{ST}_{\mathrm{NCAP}}$ is determined, the HAP transmits Ack with it to the corresponding sensor device. This operation is repeatedly conducted until $\mathrm{T}_{\text {Current }}$ reaches $\mathrm{ST}_{\mathrm{NCAP}}$. When $\mathrm{T}_{\text {Current }}$ reaches $\mathrm{ST}_{\mathrm{NCAP}}$, the notification CAP starts. 
Figure 3 shows an example of $\mathrm{L}_{\mathrm{CTAP}}$. Here, $\mathrm{L}_{\mathrm{CTAP}}$ is calculated as $\mathrm{L}_{\mathrm{PCTAP}}+\mathrm{L}_{\mathrm{DCTAP}}$, where $\mathrm{L}_{\mathrm{PCTAP}}$ is the length of the power CTAP and $\mathrm{L}_{\mathrm{DCTAP}}$ is the length of the data CTAP. LPCTAP and $\mathrm{L}_{\mathrm{DCTAP}}$ are calculated from Eqs. (1) and (2), respectively.

$$
\begin{gathered}
\mathrm{L}_{\text {PCTAP }}=\sum_{j=1}^{n} \mathrm{~L}_{\mathrm{PSLOT} j}=\sum_{i=1}^{n}\left(\mathrm{TxOffset}+\mathrm{L}_{\text {Power }}^{i}\right) \\
\mathrm{L}_{\text {DCTAP }}=\sum_{j=1}^{m} \mathrm{~L}_{\text {DSLOT } j}=\sum_{i=1}^{m}\left(\mathrm{TxOffset}+\mathrm{L}_{\text {Data }}^{i}+\mathrm{L}_{\mathrm{SIFS}}+\mathrm{L}_{\text {Ack }}\right)
\end{gathered}
$$

Here, $\mathrm{L}_{\mathrm{PSLOT}} j$ and $\mathrm{L}_{\mathrm{DSLOT}} j$ are the lengths of PSLOT $j$ and DSLOT $j$, and $\mathrm{L}_{\text {Power }}^{i}$ and $\mathrm{L}_{\text {Data }}^{i}$ are the power and data transmission times for sensor device $i$, respectively. Moreover, $\mathrm{L}_{\mathrm{SIFS}}$ is the length of the short interframe space (SIFS), $\mathrm{L}_{\text {Ack }}$ is the Ack transmission time, and $n$ and $m$ are the numbers of PSLOTs and DSLOTs, respectively. The numbers of devices requesting power charging and data delivery are equal to those of PSLOTs and DSLOTs (i.e., $n$ and $m$ ), respectively.

TxOffset, $\mathrm{L}_{\mathrm{SIFS}}$, and $\mathrm{L}_{\mathrm{Ack}}$ have fixed values. Therefore, the lengths of PSLOTs and DSLOTs depend on the power and data transmission times. For example, in Fig. 3, LPSLOT $n$ and $\mathrm{L}_{\mathrm{DSLOT} m}$ are determined from $\mathrm{L}_{\text {Power }}^{i}$ and $\mathrm{L}_{\text {Data }}^{i}$, respectively. To obtain $\mathrm{L}_{\text {Power }}^{i}$, the energy harvested by sensor device $i$ per second $\left(P_{H E}^{i}\right)$ is calculated. This is because $\mathrm{L}_{\text {Power }}^{i}$ can be obtained from the ratio of $P_{H E}^{i}$ to the charging power level of sensor device $i\left(P_{i}\right) . P_{H E}^{i}$ is given by ${ }^{(10,11)}$

$$
P_{H E}^{i}=\eta P_{T x}\left|h_{i}\right|^{2}=\eta P_{T x} /\left(1+\left|d_{i}\right|^{\alpha}\right),
$$

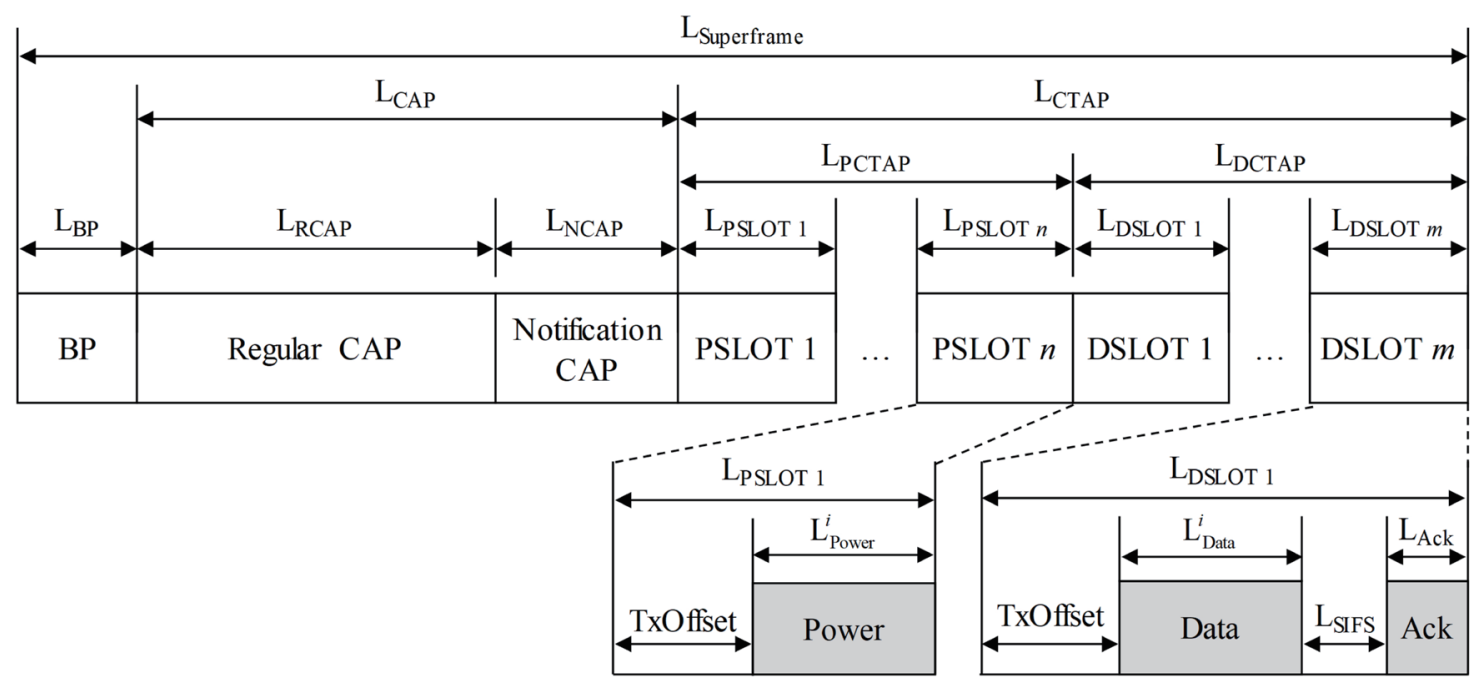

Fig. 3. Example of CTAP length. 
where $\eta$ is the efficiency factor of the EH process, $P_{T x}$ is the Tx power of the HAP, $h_{i}$ is the propagation power gain from the HAP to sensor device $i, d_{i}$ is the distance between the HAP and sensor device $i$, and $\alpha$ is the path-loss parameter. To obtain $P_{H E}^{i}, d_{i}$ is required. For this, the HAP measures the received signal strength indicator (RSSI) whenever receiving the CTAP allocation request message and estimates the distance between the device and itself. Specifically, the HAP estimates $d_{i}$ as

$$
d_{i}=10 \frac{-\mathrm{RSSI}_{i}+A}{10 \alpha},
$$

where $\operatorname{RSSI}_{i}$ is the RSSI measurement for sensor device $i$ and $A$ is the received signal strength at a distance of $1 \mathrm{~m}$. Finally, $\mathrm{L}_{\text {Power }}^{i}$ is obtained from

$$
\mathrm{L}_{\text {Power }}^{i}=P_{i} / P_{H E}^{i}=P_{i}\left(1+\left|d_{i}\right|^{\alpha}\right) / \eta P_{T x} .
$$

$\mathrm{L}_{\text {Data }}^{i}$ is simply obtained from the data size requested by sensor device $i\left(\mathrm{DS}_{i}\right)$ and the supported data rate. $\mathrm{L}_{\text {Data }}^{i}$ is given by

$$
\mathrm{L}_{\text {Data }}^{i}=\mathrm{DS}_{i} / \text { data rate } .
$$

For priority determination, the HAP adaptively assigns the PSLOT and DSLOT indices according to the residual power level of each sensor device. For this, the sensor device periodically checks its residual power level and requests power charging to the HAP when its residual power level becomes lower than the power charging threshold $\left(P_{T h}\right) . P_{T h}$ is predefined by the HAP. A low residual power level implies that power depletion is imminent. Therefore, the HAP assigns a smaller PSLOT index as the residual power level of each sensor device decreases. Similarly, in the case of data delivery, the lower the residual energy level, the smaller the DSLOT index assigned. This reduces the waiting time of sensor devices with a low residual power level; thus, data delivery failures due to power depletion can be mitigated. If there are multiple sensor devices with the same residual energy level, a smaller index is assigned to a sensor device with a lower device ID.

\section{Performance Evaluation}

We conducted an experimental simulation under an IEEE 802.11n physical layer (PHY) environment to verify the effectiveness of the hybrid MAC for TS-SWIPT using MATLAB. We compared the performance of the proposed hybrid MAC with that of the CSMA-based MAC for TS-SWIPT. In the simulation, the number of sensor devices was varied from 4 to 20 , and they transmitted the CTAP allocation request message to the HAP for power charging and data exchange during the CAP. The residual power level of each device was randomly set in 
the range of 1 to $80 \mathrm{~mW}$ and $P_{T h}$ was set to $70 \mathrm{~mW}$. Thus, the sensor devices requested power charging only when the residual power level was lower than $70 \mathrm{~mW}$. The charging power level contained in the CTAP allocation request message was determined by subtracting the residual power level from the maximum power level (i.e., $100 \mathrm{~mW}$ ). The distance between the HAP and each sensor device was randomly set from 2 to $30 \mathrm{~m}$ and the Tx power of the HAP was fixed to $100 \mathrm{~mW}$. The charging power level, distance, and Tx power were used to determine the PSLOT length of each device. The sensor devices had $1024 \mathrm{~B}$ of data to be periodically transmitted to the HAP. The data transmission interval was equal to the superframe duration, which was set to $50 \mathrm{~ms}$. The simulation was repeated 1000 times. Table 1 shows the simulation parameters in detail.

Figure 4 shows the resource utilization of the hybrid MAC for TS-SWIPT, which is the ratio of power and data transmission in one superframe (i.e., $50 \mathrm{~ms}$ ). Overall, the proposed hybrid MAC exhibits higher resource utilization than the CSMA-based MAC. This is because it allocates dedicated slots for power charging and data exchange, respectively called PSLOTs and DSLOTs, in which the sensor devices do not need to compete with each other to access the channel. In the simulation, the resource utilization of the proposed hybrid MAC is slightly lower than that of the CSMA-based MAC when the number of sensor devices is less than five. This is because the sensor device used for the CSMA-based MAC tends to be placed farther

Table 1

Simulation parameters.

\begin{tabular}{lccc}
\hline Parameter & Value & Parameter & Value \\
\hline Physical layer & IEEE $802.11 \mathrm{n}$ & MAC header & 30 bytes \\
SIFS & $10 \mu \mathrm{s}$ & Request message size & 100 bytes \\
DIFS & $50 \mu \mathrm{s}$ & Number of sensor devices & $4-20$ \\
Time slot & $20 \mu \mathrm{s}$ & Traffic rate & 20 packet/s \\
Superframe duration & $50 \mathrm{~ms}$ & Distance & $2-30 \mathrm{~m}$ \\
Data rate & $130 \mathrm{Mbps}$ & Tx power of HAP & $100 \mathrm{~mW}$ \\
TxOffset & $200 \mu \mathrm{s}$ & Charging threshold & $70 \mathrm{~mW}$ \\
Ack size & 112 bytes & Residual power level & $1-100 \mathrm{~mW}$ \\
Data size & $10 \mathrm{kbytes}$ & $\eta$ & 0.65 \\
PHY header & 16 bytes & $\alpha$ & 2.7 \\
\hline
\end{tabular}

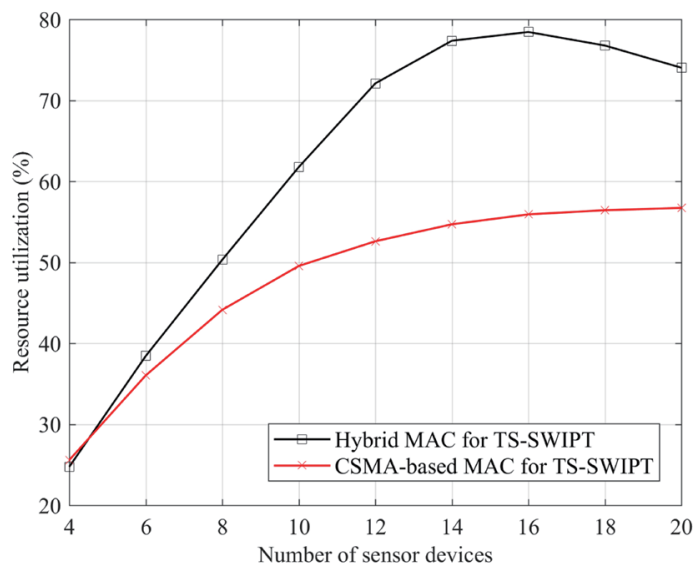

Fig. 4. (Color online) Resource utilization. 
away from the HAP than that used for the proposed hybrid MAC, resulting in a longer power delivery time. On average, the hybrid MAC for TS-SWIPT exhibits $28.9 \%$ higher resource utilization than the CSMA-based MAC for TS-SWIPT.

Figure 5 shows the aggregate throughput for different numbers of sensor devices. In the simulation, we only take the data exchange into account. In the hybrid MAC for TS-SWIPT, the sensor devices request both operations (i.e., power charging and data exchange) using one CTAP allocation request message. Therefore, the proposed hybrid MAC can mitigate the unnecessary delay caused by the individual transmission for each operation. Therefore, the hybrid MAC for TS-SWIPT has a higher aggregate throughput when the number of sensor devices increases. According to the figure, the aggregate throughput of the proposed hybrid MAC is slightly lower than that of the CSMA-based MAC. This is because, unlike the CSMAbased MAC, the proposed hybrid MAC has a notification CAP, which is used only to notify the information of CTAP allocation. However, the disadvantage of the notification CAP can be negligible since the difference in aggregate throughput between the proposed hybrid MAC and the CSMA-based MAC is very small compared with that when the number of sensor devices is more than seven. Quantitatively, the aggregate throughput of the proposed hybrid MAC is on average $21.0 \%$ higher than that of the CSMA-based MAC.

Figure 6 shows the charging delay of the sensor device with the lowest power level among the sensor devices. In the figure, the charging delay of the hybrid MAC for TS-SWIPT is shorter than that of the CSMA-based MAC when the number of sensor devices is more than five. This is because the proposed hybrid MAC determines the priority of the PSLOTs according to the residual power level of each sensor device. However, with the hybrid MAC for TS-SWIPT, the sensor devices do not conduct power charging during the CAP. In other words, the sensor devices wait until the end of the CAP, whose length is adjusted on the basis of the requested numbers and lengths of PSLOTs and DSLOTs. Therefore, the charging delay of the proposed hybrid MAC can be longer than that of the CSMA-based MAC if the number of sensor devices is small. However, the CSMA-based MAC may not guarantee the timeliness of power

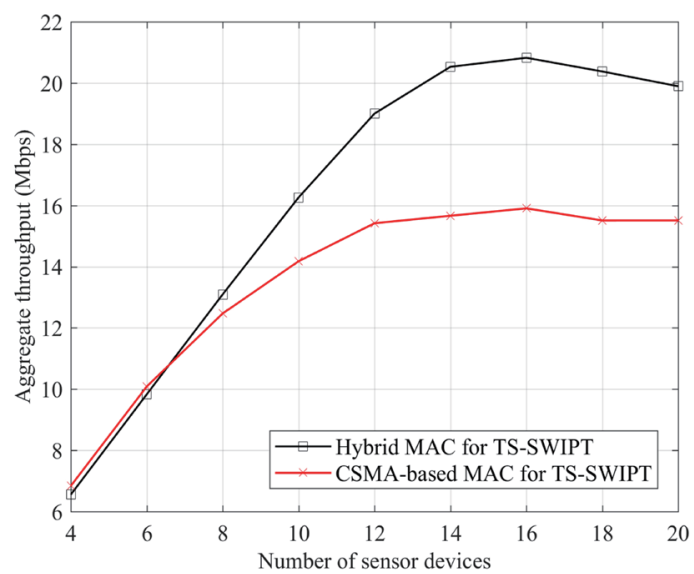

Fig. 5. (Color online) Aggregate throughput.

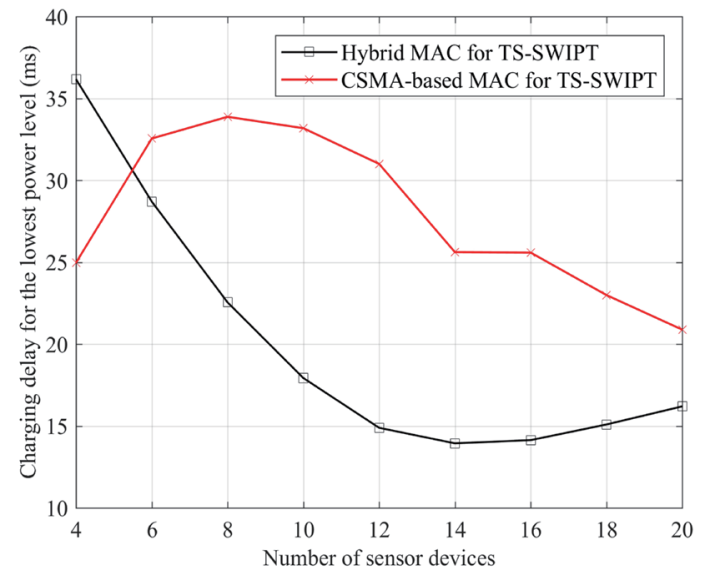

Fig. 6. (Color online) Charging delay for the lowest power level. 
delivery owing to the unpredictable delay. In the simulation, the proposed hybrid MAC exhibits $26.0 \%$ shorter charging delay than the CSMA-based MAC for the sensor devices with the lowest power level.

\section{Conclusions}

In this paper, we proposed a hybrid MAC for TS-SWIPT with the aim of improving the resource utilization and timeliness of data and power delivery. The proposed hybrid MAC uses a superframe structure consisting of a BP, a CAP, and a CTAP, and adaptively determines the length of the CAP and CTAP within the superframe by considering the number and length of the requested PSLOTs and DSLOTs. Moreover, it determines the priority of the PSLOTs and DSLOTs according to the residual power level of each sensor device. An experimental simulation was conducted to evaluate the effectiveness of the hybrid MAC for TS-SWIPT. The results show that the proposed hybrid MAC exhibits 28.9\% higher resource utilization, $21.0 \%$ higher aggregate throughput, and $26.0 \%$ shorter charging delay than the CSMA-based MAC for TS-SWIPT.

\section{Acknowledgments}

This work was supported by a Korea Evaluation Institute of Industrial Technology (KEIT) grant funded by the Korean government (MOTIE) (20001056, Development of Low-Power Massive MISO SWIPT for IoT Wearable).

\section{References}

1 F. Javed, M. K. Afzal, M. Sharif, and B.-S. Kim: IEEE Commun. Surv. Tutorials 20 (2018) 2062. https://doi. org/10.1109/COMST.2018.2817685

2 J.-H. Kwon, M. Cha, S.-B. Lee, and E.-J. Kim: Multimed. Tools Appl. 78 (2019) 2963. https://doi.org/10.1007/ s11042-017-5176-x

3 P. Porambage, J. Okwuibe, M. Liyanage, M. Ylianttila, and T. Taleb: IEEE Commun. Surv. Tutorials 20 (2018) 2961. https://doi.org/10.1109/COMST.2018.2849509

4 J.-H. Kwon, J. Park, and E.-J. Kim: Sens. Mater. 30 (2018) 1817. https://doi.org/10.18494/SAM.2018.1897

5 K. Xu, Z. Shen, Y. Wang, and X. Xia: Phys. Commun. 28 (2018) 201. https://doi.org/10.1016/j.phycom.2018.04.005

6 T. Ha, J. Kim, and J.-M. Chung: IEEE Trans. Wireless Commun. 17 (2018) 3. https://doi.org/10.1109/ TWC.2017.2757024

7 IEEE Standard for Information Technology-Telecommunications and information exchange between systems - Local and metropolitan area networks-Specific requirements - Part 11: Wireless LAN Medium Access Control (MAC) and Physical Layer (PHY) Specifications (2016) IEEE Std 802.11-2016 (Revision of IEEE Std 802.11-2012).

8 M. Y. Naderi, P. Nintanavongsa, and K. R. Chowdhury: IEEE Trans. Wireless Commun. 13 (2014) 3926. https://doi.org/10.1109/TWC.2014.2315211

9 F. Iannello, O. Simeone, and U. Spagnolini: IEEE Trans. Commun. 60 (2012) 1381. https://doi.org/10.1109/ TCOMM.2012.030712.110089

10 T. D. P. Perera, D. N. K. Jayakody, S. K. Sharma, S. Chatzinotas, and J. Li: IEEE Commun. Surv. Tutorials 20 (2018) 264. https://doi.org/10.1109/COMST.2017.2783901

11 S. He, K. Xie, W. Chen, D. Zhang, and J. Wen: IEEE Access 6 (2018) 17996. https://doi.org/10.1109/ ACCESS.2018.2820093 